\title{
Pharmacological treatment of obsessive-compulsive disorder
}

\author{
Christopher Pittenger, MD, Ph.D. and Michael H. Bloch, MD, MS \\ Department of Psychiatry and Child Study Center, Yale University School of Medicine, 34 Park \\ Street, New Haven, CT 06519 \\ Christopher Pittenger: Christopher.pittenger@yale.edu; Michael H. Bloch: Michael.bloch@yale.edu
}

\section{Synopsis}

Obsessive-compulsive disorder (OCD) affects up to $2.5 \%$ of the population of the course of a lifetime and produces substantial morbidity. Approximately $70 \%$ of patients can experience significant symptomatic relief with appropriate pharmacotherapy. The selective serotonin reuptake inhibitors (SSRIs) are the main stay of pharmacological treatment. These are typically used at higher doses and for longer periods than in depression. Remission is, unfortunately, uncommon. Proven second-line treatments include the tricyclic clomipramine and the addition of low-dose neuroleptic medications. Other augmentation strategies have been explored for patients refractory to proven interventions, but they are not as of yet robustly supported by controlled studies. The combination of medication with psychotherapy is often used, though careful studies have not documented synergistic benefit in adult patients. OCD refractory to available treatments remains a profound clinical challenge.

\section{Keywords}

Obsessive-compulsive disorder; OCD; pharmacotherapy; SSRI; antidepressant; augmentation

\section{Introduction}

Obsessive-compulsive disorder (OCD) can present a significant management challenge to the clinical psychiatrist. OCD affects approximately $1.3 \%$ of the population in any given year, and up to $2.7 \%$ over the course of a lifetime [1]. Symptoms consist of obsessions and compulsions; while either alone suffices for a diagnosis, it is typical for a patient to have both [2]. Obsessions are repetitive, stereotyped thoughts that cause anxiety or distress. These are generally experienced as intrusive or ego-dystonic, and they are typically recognized as unrealistic or excessive; this distinguishes them from delusions, although the distinction can become unclear in some severe cases. Compulsions are ritualized actions that are undertaken

(C) 2014 Elsevier Inc. All rights reserved.

Correspondence to: Christopher Pittenger, Christopher.pittenger@yale. edu.

Publisher's Disclaimer: This is a PDF file of an unedited manuscript that has been accepted for publication. As a service to our customers we are providing this early version of the manuscript. The manuscript will undergo copyediting, typesetting, and review of the resulting proof before it is published in its final citable form. Please note that during the production process errors may be discovered which could affect the content, and all legal disclaimers that apply to the journal pertain.

The authors have nothing to disclose 
to mitigate distress, often in response to obsessions. Typical obsessions and compulsions include preoccupations with contamination accompanied by repeated or ritualized washing, fear of harm to self or others accompanied by checking rituals, and a need for symmetry or order, accompanied by ordering or arranging compulsions.

OCD can be treated using pharmacotherapy, specialized psychotherapy, anatomically targeted treatments, or their combination [3]. First-line treatments include cognitivebehavioral therapy and pharmacotherapy with the selective serotonin reuptake inhibitors (SSRIs). In this article we review evidence-based pharmacotherapies for OCD, as well as alternatives that may be considered in refractory patients. Other treatment modalities are reviewed in other articles in this issue.

Unfortunately, even with optimal treatment, many patients continue to experience significant symptoms. Remission of moderate or severe OCD is uncommon, and long-term management is often necessary. The development of new, more effective treatment interventions represents an urgent clinical need.

\section{Serotonin reuptake inhibitors}

The selective serotonin reuptake inhibitors (SSRIs) are the mainstay of the pharmacological treatment of OCD. The tricyclic antidepressant clomipramine was shown to be of benefit in the early 1980s [4], but side effects limit its use as a first-line agent. Fluvoxamine was first shown to be beneficial in individuals with OCD by Goodman and colleagues in 1989 [5]. Since then, more than 20 blinded, placebo-controlled studies have firmly established the efficacy of SSRI monotherapy in OCD $[6,7]$. Because of the combination of proven efficacy and a typically benign side effect profile, SSRIs are the first-line pharmacological option for the treatment of OCD [3].

Closer examination of these studies permits several generalizations with respect to the clinical use of SSRIs for OCD. While fluvoxamine was the first SSRI shown to be efficacious and is still often thought of (and marketed) as a preferred OCD drug, there is no evidence of differential benefit among the SSRIs [7]; the choice of agent is therefore best made on the basis of side effects, drug interactions, patient preference, and similar considerations.

SSRIs are more efficacious in OCD when used at high doses, in excess of the typical dose range established by their suppliers (which are generally derived from studies of major depressive disorder). For example, doses of up to $80 \mathrm{mg}$ of fluoxetine, $40 \mathrm{mg}$ of escitalopram, $300 \mathrm{mg}$ of fluvoxamine, and $100 \mathrm{mg}$ of paroxetine are often needed; sometimes even higher doses are used [3]. The benefit of these higher doses has been clearly shown by a meta-analysis of multiple studies [8]. Interestingly, this contrasts with the use of the same agents in the treatment of major depressive disorder (MDD), in which higher doses have been shown to carry a higher side effect burden without increased benefit [9]. OCD symptoms typically also take longer to respond to SSRI monotherapy than do those of MDD; an adequate trial is 8-12 weeks [3]. The reasons for these differences between OCD and MDD response to SSRIs remain unclear. 
While these adages - higher dose and longer treatment - are widely accepted by OCD specialists, their relevance to clinical treatment of individual patients should not be overstated. The number needed to treat (NNT) for OCD patients treated with SSRI monotherapy at standard (antidepressant) doses is approximately 5, meaning that if 5 patients are treated with an SSRI, one can be expected to respond who would not have responded to placebo $[7,8]$. The NNT for a dose escalation from a medium dose to the higher doses noted above is 13-15 [8]. Therefore, while there is clear, measurable benefit to escalating SSRI dosage, the probability that it will move an individual patient from being a 'non-responder' to being a 'responder' is modest. There may, however, be smaller but still clinically meaningful improvements with dose escalation, to which studies are insensitive as they typically use a categorical definition of treatment response.

With respect to the time to response, the picture is also more complicated than the simple statement that SSRIs take 8-12 weeks to work. Some patients experience subjective improvement much more rapidly. A recent meta-analysis examining the trajectory of symptom improvement, rather than the response rate at endpoint, suggests that benefit begins in the first weeks of treatment, though it may take many weeks to become clinically (and statistically) significant in many cases [10]. Therefore, while the observation that SSRI response is typically slower in OCD than in MDD is correct, the suggestion that there is no response until after many weeks of treatment is an oversimplification.

Use of the SSRI citalopram merits particular mention. Although it is not approved by the FDA for use in OCD, citalopram is as efficacious as the SSRIs that are approved [7] and has historically been frequently used because of its generally good tolerability. However, in 2011, the FDA issued a black-box warning against the use of citalopram doses in excess of $40 \mathrm{mg} /$ day, due to a risk of ECG abnormalities and a theoretical risk of arrhythmia [11]. Doses above $20 \mathrm{mg} / \mathrm{day}$ are not recommended in the elderly, and it is recommended that the drug be avoided altogether in individuals with a $\mathrm{QT}_{\mathrm{c}}$ of greater than $500 \mathrm{msec}$ or with conditions that predispose to arrhythymia. (This effect appears to be smaller for escitalopram, which does not carry a similar FDA warning and is a reasonable alternative [12].) The merit of this warning, which did not take into account the evidence for benefit from higher doses in OCD, has been questioned [13]. Nevertheless, its existence complicates the clinical use of citalopram at high doses for OCD, and many clinicians have switched to escitalopram or another SSRI as an alternative. Because all of the SSRIs have similar efficacy, there is little reason not to make such a switch. In individual patients who have clearly benefited from citalopram and do not wish to make a switch, ECG monitoring is advisable.

\section{Children and adolescents}

The use of SSRIs in children and adolescents differs in some ways from their use in adults. Although randomized, placebo-controlled trials clearly demonstrate benefits of SSRI in children with OCD, concerns regarding side effects are more substantial [6]. The added benefit from higher doses of SSRIs, which is clear in adults, has not been demonstrated in the pediatric population. Three of the SSRIs are approved by the FDA for use in children: 
fluoxetine (age 7 and above), sertraline (age 6 and above), and fluvoxamine (age 8 and above). Clomipramine is also FDA-approved for children 10 and above.

\section{Clomipramine}

The tricyclic antidepressant clomipramine was the first agent shown clearly to be beneficial in patients with OCD [4]; it was approved by the FDA for the treatment of OCD in 1989. Of the tricyclics, clomipramine is the most potent inhibitor of serotonin reuptake. It is sometimes described as a serotonin reuptake inhibitor (SRI) - not an SSRI, because it also binds with high affinity to other receptors and reuptake sites. Meta-analysis suggests that clomipramine may be more efficacious than the SSRIs [14]. However, a variety of technical issues (such as drug dosing and the difficulty maintaining a blind in studies of clomipramine due to its side effect profile) complicate this interpretation, and head-to-head trials comparing clomipramine with SSRIs have not shown it to be superior [3]. The fact that higher doses of SSRIs are more efficacious suggests that studies using standard SSRI doses may underestimate the benefit of these agents and therefore bias a comparison with clomipramine (for which dose elevation is not possible due to the risk of cardiac toxicity). On the other hand, our recent meta-analysis suggests added benefit to clomipramine even when SSRI dosage is appropriately taken into account [10]. It seems likely that clomipramine has a small added benefit, at least for some patients.

This modestly greater efficacy must be balanced against clomipramine's more problematic side effect and safety profile. In contrast to SSRIs, clomipramine has significant anticholinergic side effects (e.g. dry mouth and constipation), anti-histaminergic effects (e.g. weight gain and sedation) and alpha-adrenergic blocking effects (e.g. hypotension). It also has substantial arrhythmogenic potential; doses at or above the upper limit of the recommended dosing range, $250 \mathrm{mg}$, may require ECG monitoring, and cardiotoxicity in overdose is a concern. Clomipramine also carries a risk of seizure at doses above $250 \mathrm{mg}$. For all of these reasons, clomipramine is not generally considered a first-line agent. It remains an important alternative when SSRI monotherapy fails [3].

One pharmacological strategy that is sometimes used is the addition of clomipramine to an SSRI, or of an SSRI to clomipramine. The motivating logic is that this may capture the benefits of clomipramine without requiring doses that produce problematic side effects. However, controlled data on the use of these strategies are sparse [15] and do not provide clear guidance as to their efficacy. The combination of clomipramine with fluvoxamine can be problematic and is best avoided. Fluvoxamine is a potent inhibitor of the liver enzyme CYP2C19 and thus inhibits the metabolism of clomipramine to desmethyl-clomipramine. This can result in marked elevations of serum clomipramine when the two agents are coadministered, raising the risk of side effects such as seizure or arrhythmia [16].

\section{Discontinuation of treatment}

Only a few studies have addressed the issue of how long to continue pharmacotherapy, once a clinical response has been achieved. This is of course a complex decision in individual cases, with benefit being weighed in the context of side effects, patient attitudes, comorbidities, the potential for drug interactions, pregnancy and lactation, and other factors. 
OCD is often a chronic condition, and remission is unfortunately rare. Treatment of an episode to remission followed by treatment discontinuation is, therefore, not a common clinical scenario.

This question can be addressed using a double-blind discontinuation study design, in which a group of stably treated patients are randomized to continue on their pharmacotherapy or be switched to a placebo. One recent study found much higher relapse rate in patients switched to placebo $(52 \%)$ than those who continued on stable escitalopram (23\%) [17]. A metaanalysis of similar studies supports this conclusion, with relapse rates in individuals switched from stable active pharmacotherapy to placebo approximately double those in patients maintained on their SSRI pharmacotherapy [18]. In general, once symptom improvement on a stable medication regimen has been achieved, these results suggest that continuation of treatment is advisable, in the absence of intolerable side effects or other case-specific factors.

\section{SNRIs}

The efficacy of clomipramine led to the hypothesis that dual-acting serotoninnorepinephrine reuptake blockers might be of greater benefit than SSRIs [19]. Indeed, an early open-label study of venlafaxine suggested that SNRIs are highly effective in the treatment of SSRI-refractory OCD, with response rates of $76 \%$ in 29 subjects [20]. However, while other uncontrolled case series have continued to suggest benefit, a subsequent double-blind crossover study suggested that venlafaxine may actually be less effective than paroxetine in the treatment of refractory OCD [21]. When 43 patients who failed to respond to one of these two agents were switched in a double-blind fashion to the alternative agent, the response rate to paroxetine (56\%) was significantly higher than that to venlafaxine (19\%) [21]. While there continues to be some theoretical rationale for the use of SNRIs, they cannot be recommended for OCD monotherapy on the basis of currently available data. Further research is needed.

\section{Pharmacological augmentation}

Monotherapy with agents beyond the SRIs has not been shown to be of benefit in OCD. When SRI monotherapy fails, therefore, pharmacological augmentation with other agents is a frequent recourse. Clear evidence exists for benefit from the addition of low-dose neuroleptics to stable SRIs. Numerous other agents have been investigated in this context, but the evidence for benefit is less clear. Nevertheless, because up to $30 \%$ of patients experience little benefit from the best evidence-based treatments, clinicians must in practice often turn to these less well-established strategies.

\section{Neuroleptic augmentation}

Double-blind trials in the late 1990s demonstrated efficacy of augmentation of SSRI pharmacotherapy with low-dose typical and atypical antipsychotics in OCD. A recent metaanalysis of 9 double-blind, placebo controlled trials of augmentation with typical or atypical antipsychotics demonstrated their efficacy compared to placebo [22, 23]. Approximately one-third of treatment refractory OCD patients will respond to antipsychotic augmentation; 
the NNT is 4.6. OCD patients with comorbid tic disorders appear to respond particularly well to antipsychotic augmentation. There is no evidence that any particular antipsychotic commonly used as augmentation is any better than any other antipsychotic agent, although the most convincing evidence for efficacy exists for haloperidol and risperidone. It should be noted that not all studies show positive results; one recent study found no benefit from the addition of risperidone to stable clomipramine treatment (whereas the addition of cognitive behavioral therapy was highly beneficial) [24]. It is clear that not all patients benefit from neuroleptic augmentation; the patients included in this recent study study had fewer tics and may have been less treatment resistant than those in earlier studies, which may explain the discrepant results.

In general, antipsychotic augementation should not be considered until two SRI trials of adequate dose and duration have been attempted, because of the more benign side effect profile of the SRIs and the reasonable likelihood of response to extended treatment or a switch to a second agent. However, if significant symptoms persist after two such trials, augmentation with a low dose of an antipsychotic represents a realistic treatment option, espeically in patients with a personal or family history of tics.

\section{Glutamatergic agents: the NMDA receptor}

Substantial recent interest has focused on the role of glutamate imbalance in OCD [25]. Polymorphisms in the gene for the major neuronal glutamate transporter have been associated with OCD, though the nature of any causative polymorphism remains unclear [26]. Several magnetic resonance spectroscopy (MRS) studies have indicated abnormalities in glutamate and related molecules, although again the specific nature of the hypothesized disruption remains unclear [27]. Finally, a pair of studies examining cerebrospinal fluid in unmedicated adults with OCD have found elevated glutamate levels [28, 29]. These findings have spurred interest in the use of gluatmate modulators for pharmacological augmentation in SRI-refractory disease; several such agents are already approved for other indications. Because these findings suggest an excess of extrasynaptic glutamate, anti-glutamatergic modulators have been the most extensively investgiated.

Agents targeting the NMDA class of glutamate receptor have received particular attention. It has been targeted in several distinct ways, which may have different effects on neuronal function and circuit dynamics. Memantine, which is used for the treatment of Alzheimer disease, is a low-affinity noncompetitive NMDA blocker. A series of small uncontrolled studies have suggested benefit in both adults and children with OCD [30-33]. One of these suggested differential benefit in OCD compared to generalized anxiety disorder, indicating that there may be some diagnostic specificity to the effect [31]. More recently, a pair of blinded, placebo controlled studies from Iran examined memantine augmentation [34] or monotherapy [35] and found a surprisingly substantial benefit. The effects reported are substantially more robust than what is suggested by the previously reported open-label studies, reaching $100 \%$ response and $89 \%$ remission after 8 weeks of treatment. These studies are promising, but replication in other populations is needed to increase confidence in the generalizability of the results. On the other hand, as memantine is an FDA-approved medication with a rather benign side effect profile at the doses used (typically $20 \mathrm{mg} / \mathrm{dy}$ ), 
emprical use in refractory patients may be reasonable even in the absence of definitive studies.

Several other studies have reported benefit from indirect modulators of the NMDA receptor. Glycine is a co-agonist of the NMDA receptor and is required for its full activation. In a small study, blinded treatment with glycine (or placebo) appeared to improve symptoms, an effect that nearly reached significance in an analysis of completers [36]. Unfortunately, glycine was very poorly tolerated, and there were many dropouts. Another small, open-label study investigated the naturally occurring amino acide analog sarcosine, which is an inhibitor of glycine reuptake and thus is predicted to indirectly increase synaptic glycine. There was a $20 \%$ reduction in OCD symptoms; this must be interpreted with caution in the absence of a placebo comparison group but is encouraging [37].

A third set of studies have used the high-potency anesthetic NMDA blocker ketamine. These are motivated by the startling observation that a single challenge with ketamine can have rapid antidepressant effects lasting days or 1-2 weeks, even in treatment-refractory MDD [38]. Two fairly small studies of ketamine challege in OCD have yielded conflicting results. In a placebo-controlled study of unmedicated patients, Rodriguez et al have shown a pattern of response very similar to what has been reported in MDD: a rapid improvement within hours of a ketamine infusion that lasts several days before symtoms return to baseline [39]. In contrast, in a somewhat more ill group of patients, many of whom had comorbidities and many of whom were medicated, Bloch and colleagues found no clinically significant benefit from ketamine [40]. This second study did not have a placebo control group, but many of the subjects were depressed, and many of those exhibited an improvement in their depressive symptoms, which therefore dissociated, from OCD subjects in the same subjects. This provides an 'internal control' documenting the adequacy of the ketamine infusion. It remains unclear whether the discrepancy between these two studies derives from methodological factors or from differences in the patient populations studied. Further research is needed. Regardless, ketamine is unlikely to become a major part of the pharmacological armamentarium: as currently administered it requires IV infusion; the effects are transient; and both basic and clinical literature raise concerns about the neurotoxic potential of chronic ketamine exposure. As in depression, therefore, any benefit from ketamine challenge is likely to be more useful as a guide to the development of future therapeutics than as a new treatment option in its own right.

It is important to note that these three ways of modulating the NMDA receptor are fundamentally different from one another. Memantine chronically blocks NMDA receptor function; in both open-label and controlled studies, benefit has been seen after weeks. Glycine and sarcosine, in contrast, are positive modulators of NMDA function and may potentiate it. Finally, ketamine is a much more potent antagonist than memantine, but its use in studies to date is fundamentally different, consisting of an acute challenge rather than chronic treatment for weeks. These very different ways of targeting the receptor are likley to have fundamentally different effects on neuronal and circuit functioning. If ongoing research corroborates the benefit of more than one of these interventiaol stratgies, an explanation for this fact will be called for. 


\section{Other glutamate-modulating agents}

A variety of other glutamate-modulating agents have been tried in OCD, chiefly in small, uncontrolled studies. A pair of open-label studies in profoundly refractory patients suggests benefit from the glutamate modulator riluzole, which is approved by the FDA for the treatment of amyotrophic lateral sclerosis [41, 42]; however, controlled data have not yet been reported. Riluzole is generally well tolerated, and some patients have been on it continuously for years without problems [42]. Riluzole has several mechanisms of action; it remains unclear which may provide benefit in OCD [43].

The antiepileptic topiramate is thought to modulate neuronal glutamate levels through its interaction with voltage-gated ion channels. Two controlled trials have suggested benefit from topiramate, at a variety of doses; there is some evidence of a greater effect on compulsions than on obsessions [44, 45]. Cognitive, sedative, and weight-loss side effects may limit the use of topiramate in some cases.

Lamotrigine is an antiepileptic and mood stabilizer. It is thought to reduce neuronal glutamate outflow through its inhibition of certain voltage-gated sodium channels, a mechanism that overlaps with some of the effects of riluzole [43]. An initial investigation of lamotrigine in OCD provided no evidence of benefit [46]. However, a more recent randomized trial investigated lamotrigine augmentation of stable SSRI treatment and found marked benefit, with 50\% responders in the lamotrigine group and none in the placebo group [47]. The reason for this discrepancy is unclear, and more work is needed.

The amino acid derivative $\mathrm{N}$-acetylcysteine has both antioxidant and glutamate-modulating properties. It has been examined in a variety of conditions and is an attractive agent because it is availaele over-the-counter, is extremely affordable, and has few side effects. An early case report suggested benefit in OCD [48]. More recently a placebo-controlled trial from Iran suggested marked benefit of the addition of N-acetycysteine to stable SSRI treatment in adults with OCD [49]. More work is needed; but the many attractive characteristics of this agent may make it a viable option in some cases once better-proven strategies have been exhausted.

\section{Other pharmacological augmentation strategies}

A variety of other agents have been used to augment ineffective SSRI treatment; none are sufficiently well supported by the literature to have entered the standard of care, but small studies provide intriguing evidence of benefit in several cases.

Mirtazapine is an a-2 adrenergic receptor antagonist and thus enhances norepinephrine release [50]; it also indirectly enhances serotonergic neurotransmission. A case series of 6 OCD patients suggested that mirtazapine is ineffective as an augmentation agent for treatment-refractory OCD [20]. However, in an unblinded trial of 49 treatment-naïve OCD patients, subjects receiving citalopram plus mirtazapine had an accelerated clinical response, with a significantly greater reduction in OCD severity at 4 weeks compared to citalopramplus-placebo. Treatment response in the two groups equalized by 12 weeks [51]. This result suggests that mirtazapine may be helpful in accelerating the initial response to SRI 
pharmacotherapy but not increasing the ultimate likelihood of response in those who fail initial SRI pharmacotherapy.

\section{Opioid augmentation}

The endogenous opioid system has been postulated to be involved in OCD pathogenesis ever since it was noted that administration of the opioid antagonist naloxone exacerbated OCD symptoms [52]. A double-blind, placebo-controlled crossover trial of 23 treatment refractory OCD patients demonstrated a significantly greater decreased in OCD symptoms in response to weekly oral morphine compared to placebo [53]. Two weeks of oral morphine produced a median decrease in Y-BOCS severity of $13 \%$, with 7 of the 23 subjects (30\%) being treatment responders [53]. Tramadol hydrochloride, an opioid agonist with lower abuse potential than morphine, has been studied as an augmentation agent for treatment refractory OCD in an open-label trial. The six treatment-refractory OCD patients included in this study experienced an average decline of Y-BOCS scores of 26\% [54]. Further doubleblind studies are needed to establish the efficacy of tramadol as an augmentation agent in OCD.

Ondansetron is a 5-HT3 receptor agonist that is used as an anti-emetic. Several small studies have suggested benefit from low doses of ondansetron and of the related agent granisetron in OCD $[55,56]$. The studies showing the clearest effects are again from Iran [57, 58]. In December of 2012, Transcept Pharmaceuticals announced that a Phase 2 trial of ondansetron had not met its primary efficacy endpoint [59]. The role, if any, for 5-HT3 agonists as augmentation in refractory disease remains to be clarified.

Caffeine was included as an active control in a pilot study of amphetamine augmentation in OCD. Both dextroamphetamine and caffeine led to significant improvements in refractory patients over 5 weeks [60]. The potential clinical use of these stimulants has not yet been followed up in larger studies.

In sum, small studies of varying quality have led to several intriguing possibilities for augmentation strategies in OCD that is refractory to standard pharmacological approaches. However, in none of these cases is such an approach supported by multiple high-quality studies. Further research is needed to address the unmet clinical needs of the substantial minority of OCD patients who do not respond to standard-of-care treatment.

\section{Over-the-counter agents}

There has been significant interest in a variety of over-the-counter (OTC) agents for OCD $[61,62]$. These are often perceived as safer and more 'natural' than prescription pharmacotherapy, which makes them attractive to many patients. $\mathrm{N}$-acetylcysteine, sarcosine, and glycine, which have been addressed above in the context of glutamate modulators, fall into this category. Unfortunately, variable quality control makes it difficult to use these less-regulated agents with confidence, and the research base guiding their use remains very thin. We have recently reviewed this literature and provide guidance for the use of OTC agents [63]. We will not extensively review the literature on these agents here but rather highlight those for which there is some substantive evidence of benefit. Most OTC 
agents are well tolerated, which is why they are lightly regulated; the typically low risk associated with their moderate use may make such a strategy reasonable, when preferred by individual patients, even when the evidence for benefit is less than robust.

Myo-inositol has been examined in two small studies. The first found evidence of benefit from monotherapy [64]. A follow-up study from the same group found no benefit from the addition of myo-inositol to stable SSRI treatment [65]. The authors suggest that this indicates an interaction, such that myo-inositol is only efficacious when used as monotherapy. However, both of these studies, while well designed, were small. More work would be needed to substantiate the use of myo-inositol to the point that it could be recommended as part of the standard of care.

\section{Other agents}

Small studies have investigated a number of other agents, including kava, St. John's wort, borage, milk thistle, eicosapentaenoic acid, and tryptophan [63]. However, for none of these is there substantial evidence of benefit for OCD symptoms (though St. John's wort may be of benefit for comorbid depressive symptoms). Potential side effects are of concern in some cases, especially with chronic use of kava.

\section{Combination treatment}

SSRI pharmacotherapy and behavioral and cognitive-behavioral psychotherapy are considered first-line treatments for OCD. (Psychotherapies for OCD are reviewed elsewhere in this issue.) It is intuitive that their combination would be more efficacious than either alone. Surprisingly, careful studies suggest that this may not always be the case, at least in an idealized setting in adults.

In the pediatric population, the POTS trial (Pediatric OCD Treatment Study) compared CBT, sertraline, and their combination with placebo in 112 children with OCD [66]. All active treatments were superior to placebo and were well tolerated. Combination treatment was more effective than either CBT alone or sertraline alone, supporting synergistic benefit. A follow-up study found that the addition of CBT to stable pharmacotherapy can provide further improvement [67]. This pair of large, definitive studies establishes rather clearly that the combination of medication and psychotherapy is more effective than either one alone, in the pediatric population.

In adults, the benefits of combination therapy are less clear. A large study comparing expert CBT, clomipramine, and their combination to placebo found that the benefit of CBT exceeded that of clomipramine, and that combination treatment provided no significant additional improvement [68]. A follow-up study from the same group looked at the addition of CBT to stable SRI treatment, compared to risperidone augmentation. CBT was markedly superior [24]. These studies suggest that combination treatment may not provide benefit.

However, important caveats to this conclusion must be noted. The CBT provided in these studies was intensive (twice weekly) and was administered by particularly skilled experts at academic centers; it is likely to be more potent than CBT as practiced in the community, 
even by experienced practitioners. Medication in these studies was administered in a manualized, relatively inflexible way, which may not recapitulate typical pharmacological strategies. In clinical practice, most authorities continue to recommend combination therapy as having potential benefits above and beyond medication or therapy alone [3]. For example, some patients cannot tolerate the anxiety that is inherent to CBT until their symptoms are somewhat moderated by medication.

\section{Augmentation of psychotherapy through enhancement of plasticity}

As our understanding of the brain processes underlying learning advances, an exciting prospect is that this knowledge can be harnessed to enhance the potency and specificity of psychotherapy. While such synergistic strategies have not entered mainstream clinical practice, there are several promising initial steps in this direction, especially in the treatment of anxiety disorders.

The NMDA glutamate receptor, described above, has a key role in modulating the strength of connections between neurons, which is thought to be a key substrate for learning. In animals, enhancement of NMDA receptor function can enhance learning [69]. This observation has led to the idea that transient enhancement of NMDA function, in conjunction with focused psychotherapeutic interventions, might lead to improved efficacy [70]. An initial proof of concept of this approach was provided by Ressler, Davis, and colleagues in a seminal set of studies. In animals, they showed that D-cycloserine, a positive allosteric modulator of the NMDA receptor, enhanced extinction of learned fear in animals [71]. They then applied this strategy to the extinction-based treatment of acrophobia, and found that D-cycloserine enhanced clinical response [72].

Several studies have sought to apply this approach to the treatment of OCD. Results to date are mixed, with some studies showing enhanced efficacy or rate of responding in patients treated with D-cycloserine prior to CBT sessions [73]. Variables such as the dosage and the timing of D-cycloserine administration are likely to be key to any benefit. The effect of Dcycloserine appears to decrease over CBT sessions, which may indicate that the primary effect is on the rate of responding, rather than on the ultimate efficacy of the treatment [73]. D-cycloserine is reasonably well tolerated and is available at compounding pharmacies; this strategy is therefore available to clinicians, though it has not entered widespread use. These findings are perhaps more valuable as a demonstration of the viability of plasticityenhancing manipulations to optimize the response to CBT; further research in this area may lead to more dramatic interactive effects.

It is important to note that the targeting of the NMDA receptor using D-cycloserine is qualitatively different from the pharmacological use of memantine, ketamine, or glycine, which have been discussed above. When D-cycloserine is used in conjunction with CBT, function of the NMDA receptor is acutely enhanced, in order to potentiate plasticity. Glycine, and similar agents, seek to chronically enhance NMDA receptor function. In contrast, ketamine transiently blocks the NMDA receptor, while memantine treatment has been used to chronically block it. The fact that such disparate therapeutic strategies targeting a single target, with some evidence of benefit for each, speaks to the centrality of this receptor in brain function and in psychopathology. 


\section{Pharmacogenetics}

There has been great excitement in recent years about pharmacogenetics: the possibility of using individual genetic data to predict drug response and/or side effects, and thus to meaningfully guide treatment choices. The appeal and theoretical potential of this approach cannot be denied. As the response to medication treatment in OCD is highly heterogeneous and the disorder has a substantial genetic component, this may be a particularly appropriate context for a pharmacogenetic approach.

In MDD, significant work has been done in this area and has implicated polymorphisms in a number of genes as predictors of antidepressant response [74, 75]. In contrast, progress in establishing genetic polymorphisms with predictive value in OCD has been relatively slow [76]. Over the past decade, our ability to gather genetic information has rapidly outstripped our knowledge of which polymorphisms are prognostically useful. Because a number of companies are now offering genotyping services to patients, it is increasingly critical for clinicians to be in a position to interpret such data.

Conceptually, there are two ways in which genetic polymorphisms may contribute to medication response. First, they could affect pharmacokinetics, by altering drug metabolism or transport; well-characterized polymorphisms in the liver's cytochrome P450 system or the blood brain barrier efflux pump are likely to fall into this category. Alternatively, polymorphisms in brain-expressed molecules related to hypothesized pathophysiological mechanisms are more likely to alter a drug's pharmacodynamics actions, either by directly altering its interactions with its molecular targets or by changing the way interacting molecules or cellular processes react to drug effects. Polymorphisms in components of the serotonergic and glutamatergic system are more likely to fall into this latter category, although it may not be possible (or necessary) to make this distinction with confidence $a$ priori.

Examination of loci in pharmacogenetic studies can be done either in a targeted, hypothesisdriven fashion, by picking genes of potential interest with known polymorphisms and examining them in the target population, or in an exploratory fashion, in which the genome is queried more broadly to find loci associated with response (i.e. pharmacogenomics). The latter approach requires many more subjects and has not yet been used in OCD.

The cytochrome P450 enzymes are highly polymorphic in the population and have a wellcharacterized role in drug metabolism, including in the metabolism in a number of drugs commonly used for the treatment of OCD. Given that polymorphisms in this system are well established to affect the rate at which different individuals metabolize specific drugs, and thus the concentration of active drug to enter the brain and the concentration of potentially active metabolites, this system represents a promising target for pharmacogenetic studies. A recent investigation examined P450 polymorphisms in 184 patients with OCD [77]. The strongest effect was an association of low-activity variants in the gene CYP2D6 with the number of past failed treatment trials in these patients, which may be a surrogate marker for refractoriness. There was also a lower incidence of side effects from venlafaxine in individuals with normal ('extensive') CYP2D6 metabolic activity than those with lower 
metabolizing alleles. This may be due to the fact that venlafaxine is metabolized to an active metabolite, O-desmethylvenlafaxine, which is therefore reduced in low-metabolizers. Trendlevel effects suggested an influence of CYP2D6 metabolizer status on response to fluoxetine and of CYP2C19 status on response to sertraline. These findings require replication and do not yet provide clear guidance for the application of genetic data to treatment selection, but they represent a promising start to a potentially important new source of information to guide clinical decision-making.

Other small studies in OCD have examined polymorphisms in genes more likely to be associated with pharmacodynamics, such as the serotonin receptor HTR2A, the serotonin transporter SLC6A4, the neurotransmitter BDNF, and the monoamine metabolic enzyme COMT. However, these studies have by and large been small and inconsistent [76]. More work is needed in this area.

\title{
Conclusion
}

Our treatments for OCD remain inadequate. While a majority of patients will respond to established pharmacotherapy and/or psychotherapeutic approaches, approximately a quarter do not. Furthermore, many of those who are classified as 'responders' continue to have substantial symptoms and a chronic reduction in productivity and in quality of life.

The SSRIs are the mainstay of the pharmacological treatment of OCD. Their combination of efficacy with relatively good tolerability is not matched by any other available agents. There is no known difference in the efficacy of different SSRIs, and thus the choice of agent is best guided by side effects, pharmacokinetic considerations, and patient preference. Higher doses and longer duration of treatment, relative to standard practice in the treatment of MDD, is often required. Clomipramine provides an alternative for monotherapy and may be marginally more effective, but its side effect profile mitigates against its use as a first-line agent in most cases. When monotherapy fails, augmentation with low-dose neuroleptic (especially risperidone) or psychotherapy has good support in the literature. Other augmentation strategies are less well established but are often appropriate when first- and second-line approaches have been exhausted.

Perhaps the most important conclusion from this review of pharmacotherapeutic options is that there is a relative paucity of well-established treatment options in OCD, when SSRI treatment fails. This contrasts with MDD, schizophrenia, and many other major psychiatric conditions, in which numerous mechanistically distinct pharmacological strategies are available and algorithms for stepped treatment are being developed. It is to be hoped that, as more research is done in the pathophysiology and treatment of OCD and related disorders, clinicians will have a broader array of treatment options in the future.

\section{Key abbreviations}

\author{
OCD obsessive compulsive disorder \\ SRI serotonin reuptake inhibitor
}


SSRI selective serotonin reuptake inhibitor

TS Tourette syndrome

MDD major depressive disorder

\section{References}

1. Kessler RC, et al. Twelve-month and lifetime prevalence and lifetime morbid risk of anxiety and mood disorders in the United States. Int J Methods Psychiatr Res. 2012; 21(3):169-184. [PubMed: 22865617]

2. American Psychiatric Association. and American Psychiatric Association. Diagnostic and statistical manual of mental disorders : DSM-5. 5th ed.. Washington, DC: American Psychiatric Association; 2013. DSM-5 Task Force; p. xlivp. 947

3. Koran LM, et al. Practice guideline for the treatment of patients with obsessive-compulsive disorder. Am J Psychiatry. 2007; 164(7 Suppl):5-53. [PubMed: 17849776]

4. Insel TR, et al. Obsessive-compulsive disorder. A double-blind trial of clomipramine and clorgyline. Arch Gen Psychiatry. 1983; 40(6):605-612. [PubMed: 6342562]

5. Goodman WK, et al. Efficacy of fluvoxamine in obsessive-compulsive disorder. A double-blind comparison with placebo. Arch Gen Psychiatry. 1989; 46(1):36-44. [PubMed: 2491940]

6. Soomro GM. Obsessive compulsive disorder. Clin Evid (Online). 2012; 2012

7. Soomro GM, et al. Selective serotonin re-uptake inhibitors (SSRIs) versus placebo for obsessive compulsive disorder (OCD). Cochrane Database Syst Rev. 2008; (1):CD001765. [PubMed: 18253995]

8. Bloch MH, et al. Meta-analysis of the dose-response relationship of SSRI in obsessive-compulsive disorder. Mol Psychiatry. 2010; 15(8):850-855. [PubMed: 19468281]

9. Bollini P, et al. Effectiveness of antidepressants. Meta-analysis of dose-effect relationships in randomised clinical trials. Br J Psychiatry. 1999; 174:297-303. [PubMed: 10533547]

10. Issari Y, et al. Meta-analysis: early onset of selective serotonin reuptake inhibotors and clomipramine in obsessive-compulsive disorder. submitted.

11. FDA. FDA Drug Safety Comunications: Revised recommendations for Celexa (citalopram hydrobromide) related to a potential risk of abnormal heart rhythms with high doses. 2012

12. Therapeutics, M.L.o.D.a. Citalopram, escitalopram, and the QT interval. Med Lett Drugs Ther. 2013; 55(1421):59.

13. Zivin K, et al. Safety of high-dosage citalopram. Am J Psychiatry. 2014; 171(1):20-22. [PubMed: 24399424]

14. Ackerman DL, Greenland S. Multivariate meta-analysis of controlled drug studies for obsessivecompulsive disorder. J Clin Psychopharmacol. 2002; 22(3):309-317. [PubMed: 12006902]

15. Marazziti D, et al. Effectiveness of long-term augmentation with citalopram to clomipramine in treatment-resistant OCD patients. CNS Spectr. 2008; 13(11):971-976. [PubMed: 19037176]

16. Szegedi A, et al. Combination treatment with clomipramine and fluvoxamine: drug monitoring, safety, and tolerability data. J Clin Psychiatry. 1996; 57(6):257-264. [PubMed: 8666564]

17. Fineberg NA, et al. Escitalopram prevents relapse of obsessive-compulsive disorder. Eur Neuropsychopharmacol. 2007; 17(6-7):430-439. [PubMed: 17240120]

18. Donovan MR, et al. Comparative efficacy of antidepressants in preventing relapse in anxiety disorders - a meta-analysis. J Affect Disord. 2010; 123(1-3):9-16. [PubMed: 19616306]

19. Dell'Osso B, et al. Serotonin-norepinephrine reuptake inhibitors in the treatment of obsessivecompulsive disorder: A critical review. J Clin Psychiatry. 2006; 67(4):600-610. [PubMed: 16669725]

20. Hollander E, et al. Venlafaxine in treatment-resistant obsessive-compulsive disorder. J Clin Psychiatry. 2003; 64(5):546-550. [PubMed: 12755657] 
21. Denys D, et al. A double-blind switch study of paroxetine and venlafaxine in obsessive-compulsive disorder. J Clin Psychiatry. 2004; 65(1):37-43. [PubMed: 14744166]

22. Bloch MH, et al. A systematic review: antipsychotic augmentation with treatment refractory obsessive-compulsive disorder. Mol Psychiatry. 2006; 11(7):622-632. [PubMed: 16585942]

23. Dold M, et al. Antipsychotic augmentation of serotonin reuptake inhibitors in treatment-resistant obsessive-compulsive disorder: a meta-analysis of double-blind, randomized, placebo-controlled trials. Int J Neuropsychopharmacol. 2013; 16(3):557-574. [PubMed: 22932229]

24. Simpson HB, et al. Cognitive-behavioral therapy vs risperidone for augmenting serotonin reuptake inhibitors in obsessive-compulsive disorder: a randomized clinical trial. JAMA Psychiatry. 2013; 70(11):1190-1199. [PubMed: 24026523]

25. Pittenger C, Bloch MH, Williams K. Glutamate abnormalities in obsessive compulsive disorder: neurobiology, pathophysiology, and treatment. Pharmacol Ther. 2011; 132(3):314-332. [PubMed: 21963369]

26. Stewart SE, et al. Meta-analysis of association between obsessive-compulsive disorder and the 3' region of neuronal glutamate transporter gene SLC1A1. Am J Med Genet B Neuropsychiatr Genet. 2013; 162B(4):367-379. [PubMed: 23606572]

27. Brennan BP, et al. A critical review of magnetic resonance spectroscopy studies of obsessivecompulsive disorder. Biol Psychiatry. 2013; 73(1):24-31. [PubMed: 22831979]

28. Bhattacharyya $\mathrm{S}$, et al. Anti-brain autoantibodies and altered excitatory neurotransmitters in obsessive-compulsive disorder. Neuropsychopharmacology. 2009; 34(12):2489-2496. [PubMed: 19675532]

29. Chakrabarty K, et al. Glutamatergic dysfunction in OCD. Neuropsychopharmacology. 2005; 30(9): 1735-1740. [PubMed: 15841109]

30. Pasquini M, Biondi M. Memantine augmentation for refractory obsessive-compulsive disorder. Prog Neuropsychopharmacol Biol Psychiatry. 2006; 30(6):1173-1175. [PubMed: 16730870]

31. Poyurovsky M, et al. Memantine for treatment-resistant OCD. Am J Psychiatry. 2005; 162(11): 2191-2192. [PubMed: 16263867]

32. Stewart SE, et al. A single-blinded case-control study of memantine in severe obsessivecompulsive disorder. J Clin Psychopharmacol. 2010; 30(1):34-39. [PubMed: 20075645]

33. Feusner JD, et al. Differential efficacy of memantine for obsessive-compulsive disorder vs. generalized anxiety disorder: an open-label trial. Psychopharmacol Bull. 2009; 42(1):81-93. [PubMed: 19204653]

34. Ghaleiha A, et al. Memantine add-on in moderate to severe obsessive-compulsive disorder: randomized double-blind placebo-controlled study. J Psychiatr Res. 2013; 47(2):175-180. [PubMed: 23063327]

35. Haghighi M, et al. In a double-blind, randomized and placebo-controlled trial, adjuvant memantine improved symptoms in inpatients suffering from refractory obsessive-compulsive disorders (OCD). Psychopharmacology (Berl). 2013; 228(4):633-640. [PubMed: 23525525]

36. Greenberg WM, et al. Adjunctive glycine in the treatment of obsessive-compulsive disorder in adults. J Psychiatr Res. 2009; 43(6):664-670. [PubMed: 19046587]

37. Wu PL, et al. Sarcosine therapy for obsessive compulsive disorder: a prospective, open-label study. J Clin Psychopharmacol. 2011; 31(3):369-374. [PubMed: 21508860]

38. Krystal JH, Sanacora G, Duman RS. Rapid-acting glutamatergic antidepressants: the path to ketamine and beyond. Biol Psychiatry. 2013; 73(12):1133-1141. [PubMed: 23726151]

39. Rodriguez CI, et al. Randomized Controlled Crossover Trial of Ketamine in ObsessiveCompulsive Disorder: Proof-of-Concept. Neuropsychopharmacology. 2013

40. Bloch MH, et al. Effects of ketamine in treatment-refractory obsessive-compulsive disorder. Biol Psychiatry. 2012; 72(11):964-970. [PubMed: 22784486]

41. Coric V, et al. Riluzole augmentation in treatment-resistant obsessive-compulsive disorder: an open-label trial. Biol Psychiatry. 2005; 58(5):424-428. [PubMed: 15993857]

42. Pittenger $\mathrm{C}$, et al. Riluzole augmentation in treatment-refractory obsessive-compulsive disorder: a series of 13 cases, with long-term follow-up. J Clin Psychopharmacol. 2008; 28(3):363-367.

[PubMed: 18480706] 
43. Pittenger C, et al. Riluzole in the treatment of mood and anxiety disorders. CNS Drugs. 2008; 22(9):761-786. [PubMed: 18698875]

44. Berlin HA, et al. Double-blind, placebo-controlled trial of topiramate augmentation in treatmentresistant obsessive-compulsive disorder. J Clin Psychiatry. 2011; 72(5):716-721. [PubMed: 20816027]

45. Mowla A, et al. Topiramate Augmentation in Resistant OCD: A Double-Blind Placebo-Controlled Clinical Trial. CNS Spectr. 2010

46. Kumar TC, Khanna S. Lamotrigine augmentation of serotonin re-uptake inhibitors in obsessivecompulsive disorder. Aust N Z J Psychiatry. 2000; 34(3):527-528. [PubMed: 10881981]

47. Bruno A, et al. Lamotrigine augmentation of serotonin reuptake inhibitors in treatment-resistant obsessive-compulsive disorder: a double-blind, placebo-controlled study. J Psychopharmacol. 2012; 26(11):1456-1462. [PubMed: 22351381]

48. Lafleur DL, et al. N-acetylcysteine augmentation in serotonin reuptake inhibitor refractory obsessive-compulsive disorder. Psychopharmacology (Berl). 2006; 184(2):254-256. [PubMed: 16374600]

49. Afshar $\mathrm{H}$, et al. $\mathrm{N}$-acetylcysteine add-on treatment in refractory obsessive-compulsive disorder: a randomized, double-blind, placebo-controlled trial. J Clin Psychopharmacol. 2012; 32(6):797-803. [PubMed: 23131885]

50. de Boer T. The pharmacologic profile of mirtazapine. J Clin Psychiatry. 1996; 57(Suppl 4):19-25. [PubMed: 8636062]

51. Pallanti S, Quercioliand L, Koran LM. Citalopram intravenous infusion in resistant obsessivecompulsive disorder: an open trial. J Clin Psychiatry. 2002; 63(9):796-801. [PubMed: 12363120]

52. Insel TR, Pickar D. Naloxone administration in obsessive-compulsive disorder: report of two cases. Am J Psychiatry. 1983; 140(9):1219-1220. [PubMed: 6614234]

53. Koran LM, et al. Double-blind treatment with oral morphine in treatment-resistant obsessivecompulsive disorder. J Clin Psychiatry. 2005; 66(3):353-359. [PubMed: 15766302]

54. Shapira NA, et al. Open-label pilot study of tramadol hydrochloride in treatment-refractory obsessive-compulsive disorder. Depress Anxiety. 1997; 6(4):170-173. [PubMed: 9559288]

55. Pallanti S, et al. Ondansetron augmentation in treatment-resistant obsessive-compulsive disorder: a preliminary, single-blind, prospective study. CNS Drugs. 2009; 23(12):1047-1055. [PubMed: 19958042]

56. Pallanti S, et al. Ondansetron augmentation in patients with obsessive-compulsive disorder who are inadequate responders to serotonin reuptake inhibitors: Improvement with treatment and worsening following discontinuation. Eur Neuropsychopharmacol. 2013

57. Askari N, et al. Granisetron adjunct to fluvoxamine for moderate to severe obsessive-compulsive disorder: a randomized, double-blind, placebo-controlled trial. CNS Drugs. 2012; 26(10):883-892. [PubMed: 22873680]

58. Soltani F, et al. A double-blind, placebo-controlled pilot study of ondansetron for patients with obsessive-compulsive disorder. Hum Psychopharmacol. 2010; 25(6):509-513. [PubMed: 20737524]

59. Transcept Pharmaceuticals, I. Transcept Pharmaceuticals announces that a Phase 2 clinical trial of $\mathrm{TO}=2061$ as adjunctive therapy for obsessive-compulsive disorder did not meet primary endpoint. 2012 Available from: http://ir.transcept.com/releasedetail.cfm?ReleaseID=728327.

60. Koran LM, Aboujaoude E, Gamel NN. Double-blind study of dextroamphetamine versus caffeine augmentation for treatment-resistant obsessive-compulsive disorder. J Clin Psychiatry. 2009; 70(11):1530-1535. [PubMed: 19573497]

61. Sarris J, Camfield D, Berk M. Complementary medicine, self-help, and lifestyle interventions for obsessive compulsive disorder (OCD) and the OCD spectrum: a systematic review. J Affect Disord. 2012; 138(3):213-221. [PubMed: 21620478]

62. Camfield DA, Sarris J, Berk M. Nutraceuticals in the treatment of obsessive compulsive disorder (OCD): a review of mechanistic and clinical evidence. Prog Neuropsychopharmacol Biol Psychiatry. 2011; 35(4):887-895. [PubMed: 21352883] 
63. Kichuk SA, Carlton RM, Pittenger C. Over-the-counter supplements in the treatment of obsessive compulsive disorder: practical considerations and evidence. Newsletter of the International OCD Foundation. 2013:17-20. Summer 2013.

64. Fux M, et al. Inositol treatment of obsessive-compulsive disorder. Am J Psychiatry. 1996; 153(9): 1219-1221. [PubMed: 8780431]

65. Fux M, Benjamin J, Belmaker RH. Inositol versus placebo augmentation of serotonin reuptake inhibitors in the treatment of obsessive-compulsive disorder: a double-blind cross-over study. Int $\mathbf{J}$ Neuropsychopharmacol. 1999; 2(3):193-195. [PubMed: 11281989]

66. Pediatric, O.C.D.T.S.T. Cognitive-behavior therapy, sertraline, and their combination for children and adolescents with obsessive-compulsive disorder: the Pediatric OCD Treatment Study (POTS) randomized controlled trial. JAMA. 2004; 292(16):1969-1976. [PubMed: 15507582]

67. Franklin ME, et al. Cognitive behavior therapy augmentation of pharmacotherapy in pediatric obsessive-compulsive disorder: the Pediatric OCD Treatment Study II (POTS II) randomized controlled trial. JAMA. 2011; 306(11):1224-1232. [PubMed: 21934055]

68. Foa EB, et al. Randomized, placebo-controlled trial of exposure and ritual prevention, clomipramine, and their combination in the treatment of obsessive-compulsive disorder. Am J Psychiatry. 2005; 162(1):151-161. [PubMed: 15625214]

69. Myers KM, Carlezon WA Jr, Davis M. Glutamate receptors in extinction and extinction-based therapies for psychiatric illness. Neuropsychopharmacology. 2011; 36(1):274-293. [PubMed: 20631689]

70. Krystal JH, et al. Neuroplasticity as a target for the pharmacotherapy of anxiety disorders, mood disorders, and schizophrenia. Drug Discov Today. 2009; 14(13-14):690-697. [PubMed: 19460458]

71. Walker DL, et al. Facilitation of conditioned fear extinction by systemic administration or intraamygdala infusions of D-cycloserine as assessed with fear-potentiated startle in rats. J Neurosci. 2002; 22(6):2343-2351. [PubMed: 11896173]

72. Ressler KJ, et al. Cognitive enhancers as adjuncts to psychotherapy: use of Dcycloserine in phobic individuals to facilitate extinction of fear. Arch Gen Psychiatry. 2004; 61(11):1136-1144. [PubMed: 15520361]

73. Norberg MM, Krystal JH, Tolin DF. A meta-analysis of D-cycloserine and the facilitation of fear extinction and exposure therapy. Biol Psychiatry. 2008; 63(12):1118-1126. [PubMed: 18313643]

74. Gvozdic K, et al. Genetics and personalized medicine in antidepressant treatment. Curr Pharm Des. 2012; 18(36):5853-5878. [PubMed: 22681166]

75. Kato M, Serretti A. Review and meta-analysis of antidepressant pharmacogenetic findings in major depressive disorder. Mol Psychiatry. 2010; 15(5):473-500. [PubMed: 18982004]

76. Brandl EJ, Muller DJ, Richter MA. Pharmacogenetics of obsessivecompulsive disorders. Pharmacogenomics. 2012; 13(1):71-81. [PubMed: 22176623]

77. Brandl EJ, et al. Influence of CYP2D6 and CYP2C19 gene variants on antidepressant response in obsessive-compulsive disorder. Pharmacogenomics J. 2013 


\section{Key Points}

- A majority of cases of OCD will improve with appropriate pharmacotherapy.

- The mainstay of pharmacotherapy is the use of selective serotonin reuptake inhibitors (SSRIs)

- Second-line options include clomipramine and augmentation with neuroleptics

- A substantial minority of patients remain refractory to aggressive pharmacotherapy 